ISSN 0103-5150

Fisioter. Mov., Curitiba, v. 25, n. 3, p. 629-637, jul./set. 2012 Licenciado sob uma Licença Creative Commons

\title{
Análise da radiografia de tórax de indivíduos com DPOC e sua correlação com os testes funcionais
}

\author{
Analysis of chest radiography of individuals with \\ COPD and its correlation with functional testing
}

\section{Leilane Marcos ${ }^{[a]}$, Gerson Linck Bichinho ${ }^{[b]}$, Emmanuel Alvarenga Panizzi ${ }^{[c]}$, Keidy Karla Gonçalves Storino ${ }^{[\mathrm{d}]}$, Davi Carpintéro Pinto ${ }^{[\mathrm{e}]}$}

[a] Mestre em Tecnologia em Saúde pela Pontifícia Universidade Católica do Paraná (PUCPR), docente do Departamento de Fisioterapia do Centro Universitário Leonardo da Vinci, Blumenau, SC - Brasil, e-mail: fisio_lane@yahoo.com.br

[b] Doutor em Genie Biomedicale pela Universite de Technologie de Compiegne (França), professor titular e docente do curso de Pós-Graduação em Tecnologia em Saúde, da Pontifícia Universidade Católica do Paraná (PUCPR), Curitiba, PR - Brasil, e-mail: gerson.bichinho@pucpr.br

[c] Mestre em Tecnologia em Saúde pela Pontifícia Universidade Católica do Paraná (PUCPR), docente do curso de Fisioterapia da Univali, Itajaí, SC - Brasil, e-mail: panizzi@univali.br

[d] Especialista em Fisioterapia Cardiorrespiratória pela CESUMAR, fisioterapeuta da Unimed Litoral, Itajaí, SC - Brasil, e-mail: keidy.goncalves@unimedlitoral.com.br

[e] Especialista em Fisioterapia Cardiorrespiratória pela CESUMAR, fisioterapeuta da Unimed Litoral, Itajaí, SC - Brasil, e-mail: davi.pinto@unimedlitoral.com.br

\section{Resumo}

Introdução: A doença pulmonar obstrutiva crônica (DPOC) provoca alterações na função respiratória e altera as estruturas musculoesqueléticas da caixa torácica, limitando, cada vez mais, a capacidade de respiração do indivíduo. Objetivos: analisar quantitativamente as radiografias torácicas de indivíduos com DPOC e estabelecer um comparativo entre a prova de função pulmonar, a força muscular respiratória, a mobilidade torácica, a capacidade funcional e os achados dos exames radiográficos. Método: Foram avaliados 15 indivíduos com DPOC, os quais realizaram os exames de espirometria, teste da caminhada de seis minutos, manovacuometria e cirtometria. Os valores obtidos nesses testes foram comparados com medidas efetuadas na radiografia de tórax desses pacientes. Os valores obtidos para cada variável foram normalizados dividindo-se tais valores pela altura retroesternal. Resultados: A variável que apresentou correlação mais significativa com as variáveis de espirometria (CVF e PFE) e de cirtometria (Xifoide CR e Axilar CR) foi o 
ângulo costofrênico. A variável de imagem ALB DIR correlacionou-se positivamente com a variável PI máx. O nível de rebaixamento diafragmático esquerdo e a distância intercostal direita e esquerda não apresentaram correlação com as variáveis dos exames fisioterápicos. Conclusão: A variável que apresentou correlação mais significativa com os exames foi o ângulo costofrênico, o que indica que o comprometimento do diafragma impacta nas condições gerais do DPOC. Sendo assim, o estudo apontou que o comprometimento do músculo diafragma observado nos exames de fisioterapia em indivíduos com DPOC correlaciona-se com as variáveis de imagem, que avaliam o diafragma nas imagens radiográficas.

Palavras-chave: Doença pulmonar obstrutiva crônica. Avaliação. Radiografias.

\section{Abstract}

Introduction: Chronic obstructive pulmonary disease (COPD) causes changes in respiratory function and alters the musculoskeletal structures of the chest, increasingly limiting an individual's ability to breathe. Objectives: To quantitatively analyze chest radiographs of patients with COPD and establish a comparison among the pulmonary function, respiratory muscle strength, thoracic mobility, functional capacity and findings of radiographic exams. Method: We studied 15 subjects with COPD who performed the spirometry test, the six-minute walk, manovacuometry and micrometry. The values obtained from these tests were compared with measurements made on chest radiographs of these patients. The values obtained for each variable were normalized by dividing them by retrosternal height. Results: The variable that correlated most significantly with the variables of spirometry (FVC and PEF) and cirtometry (Xiphoid CR and Axillary CR) was the costo-phrenic angle. The image variable ALB DIR positively correlated with the variable PI max. The level of left diaphragmatic drawdown, intercostals right and left distances were not correlated with the variables of physiotherapy exams. Conclusion: The variable that correlated most significantly with the tests was the costo-phrenic angle, which indicates that the involvement of the diaphragm has impact on the general conditions of COPD. Thus, the study showed that impairment of the diaphragm in patients with COPD observed in tests of physiotherapy correlate with image variables that assess the diaphragm in radiography.

Keywords: Chronic obstructive pulmonary disease. Evaluation. Radiography.

\section{Introdução}

A doença pulmonar obstrutiva crônica é definida como uma enfermidade que se caracteriza pela presença de obstrução ou limitação crônica do fluxo aéreo, apresentando progressão lenta e irreversível. Tais alterações se originam da combinação de bronquite crônica com enfisema pulmonar, sendo que a predominância de um ou outro componente é extremamente variável e difícil de quantificar (1).

A DPOC apresenta manifestações fisiopatológicas que acometem pequenas e grandes vias aéreas, parênquima pulmonar, vascularização pulmonar e a musculatura respiratória, em uma combinação altamente variável de paciente para paciente (1-3).

É necessária uma avaliação criteriosa das alterações provocadas pela DPOC, recolhendo dados de exames clínicos, exames laboratoriais e estudos de imagem para, assim, elaborar o plano de tratamento para os portadores da doença. Além disso, serve para reavaliar a aplicabilidade do tratamento proposto e alterá-lo, se necessário. Esse controle da aplicabilidade das técnicas busca atestar a qualidade dos tratamentos e assegurar resultados positivos para o indivíduo (4).

A avaliação radiográfica do tórax, embora seja um exame comumente realizado em DPOC, não impacta no diagnóstico da doença. 0 exame é realizado para descartar outros diagnósticos, pois não apresenta dados quantitativos e sua avaliação depende da observação da imagem, que, por sua vez, é influenciada pelo conhecimento e pela experiência de cada profissional. Um dos sinais mais evidentes nas radiografias é a hiperinsuflação, alteração decorrente da destruição das fibras elásticas $(1,5)$.

A hiperinsuflação leva a alterações na musculatura pulmonar, principalmente para a incursão do diafragma, alterando a disposição da parede torácica $(6,7)$. 
Embora existam muitos métodos para avaliar os diferentes sistemas que a DPOC modifica, as alterações provocadas na caixa torácica são pouco abordadas pela literatura. Como a fisioterapia tem seu olhar voltado para a função, avaliar e mensurar as alterações musculoesqueléticas pode apontar melhores formas de tratamento fisioterápico, objetivando a melhoria da qualidade de vida desses indivíduos.

A radiografia de tórax, um exame de baixo custo, pode apontar dados mais específicos da influência das alterações provenientes da doença na caixa torácica e, consequentemente, na função pulmonar. Para tanto, é necessário que esse exame indique as alterações de caixa torácica com dados quantitativos em relação ao impacto da DPOC, para que, assim, seja possível monitorar e traçar de maneira mais adequada os objetivos do tratamento realizado pela fisioterapia (8).

\section{Materiais e métodos}

A amostra foi composta por 15 indivíduos com DPOC, selecionados com os seguintes critérios de inclusão: (a) assinatura do termo de consentimento livre e esclarecido; (b) apresentação de diagnóstico clínico de DPOC, de moderado a muito grave; (c) funções neurocognitivas preservadas; e (d) apresentação de imagens radiográficas de boa qualidade. Como critérios de exclusão, definiu-se: (a) não apresentar doença cardíaca grave associada; e (b) radiografias de qualidade inferior, que impossibilitassem a análise.

O estudo obteve a aprovação do Comitê de Ética da PUCPR, sob Protocolo número 0002576/09 e Protocolo CONEP 0040.0.084.000-09.

Para os exames de espirometria, teste da caminhada de seis minutos, cirtometria e manovacuometria, foram estabelecidos alguns critérios de execução da avaliação. A avaliação espirométrica foi realizada de acordo com técnica e valores de referência padronizados pela American Toracic Society (ATS) e pelas Diretrizes para Testes de Função Pulmonar (SBPT) $(9,10)$. As variáveis analisadas foram: capacidade vital forçada (CVF); volume expiratório forçado no primeiro segundo $\left(\mathrm{VEF}_{1}\right)$; pico de fluxo expiratório forçado (PEF); e índice de Tiffeneau ( $\mathrm{VEF}_{1} / \mathrm{CVF}$ ). Para que o exame seja aceito, ele deve responder a critérios de reprodutibilidade: selecionar a maior CVF, a maior VEF das curvas com valores de PFE aceitáveis e os dois maiores valores de VEF1 e CVF, que devem diferir menos de 0,15 litros.
0 teste de caminhada de seis minutos foi realizado com a supervisão de um profissional, sendo que no início e no fim do exame foram coletados dados de pressão arterial, frequência cardíaca, saturação e dispneia, por meio da Escala de Borg. Os indivíduos foram instruídos a caminharem no corredor de 30 metros de comprimento, delimitado por dois cones, tentando alcançar a maior distância possível num intervalo de tempo de seis minutos. Foi anotada a distância máxima percorrida entre os cones $(11,12)$.

Para a realização da cirtometria, utilizou-se uma fita métrica, marca Cateb, com escala de 0 a 150 centímetros e precisão de 0,1 centímetro, medindo os perímetros torácicos em três regiões: perímetro axilar, perímetro xifoide e perímetro basal. A medida foi realizada na inspiração máxima da capacidade pulmonar total e, posteriormente, na expiração máxima ao nível do volume residual, nas três regiões citadas anteriormente. A diferença entre as duas medidas (da inspiração e da expiração) é denominada de coeficiente respiratório $(\mathrm{Cr})$, representando a mobilidade torácica $(13,14)$.

$\mathrm{Na}$ avaliação da força muscular respiratória por meio da manovacuometria, foram obtidos valores de pressão inspiratória máxima (refletindo a função dos músculos inspiratórios) e de pressão expiratória máxima (função dos músculos expiratórios). A execução da manobra de PI máx consiste na expiração até o volume residual e a realização de um esforço inspiratório máximo por três segundos. A PE máx exige uma inspiração próxima à capacidade pulmonar total e a execução de esforço expiratório máximo $(15,16,17)$.

Os dados obtidos nesses testes foram associados às variáveis avaliadas na radiografia. Para essa avaliação, os exames radiográficos do tórax foram realizados na posição posteroanterior (PA) e processados no Laboratório de Engenharia da Reabilitação (LER) da PUCPR, apresentando 256 níveis de cinza e resolução de 150 pixels/polegadas.

Todas as medidas efetuadas nas imagens foram obtidas com a ajuda de um software desenvolvido em MatLab ${ }^{\circledR}$. 0 software possibilitou a obtenção das medidas em pixels, que foram salvas em um arquivo Excel ${ }^{\circledR}$ para posterior análise (Figura 1).

As variáveis consideradas para análise foram: (a) altura retroesternal pulmonar (AREP), do início ao fim do pulmão direito, pois o pulmão esquerdo sofre influência da área cardíaca; (b) largura pulmonar (LARGP) entre o sexto e o sétimo espaço intercostal, pois é um ponto mediano do gradil costal descrito na 
literatura; (c) nível de rebaixamento diafragmático direito e esquerdo (ALBDIR e ALBESQ), subdivido em altura da base do diafragma do lado direito - linha de base do diafragma até o ângulo costocárdico direito - e em altura da base do diafragma do lado esquerdo - linha de base do diafragma até o ângulo costocárdico esquerdo; (d) ângulo costofrênico ou costodiafragmático (ANGCF), entre a linha do ângulo e a linha da base do diafragma; (e) distância intercostal direita e esquerda (DISDIR e DISESQ), medida para avaliar a presença do aumento dos espaços intercostais. Para podermos comparar os valores obtidos (em pixels) pelas imagens de indivíduos diferentes, é necessário efetuar a normalização dos valores. Assim sendo, a normalização das variáveis foi realizada dividindo-se os valores das variáveis pela altura retroesternal. A altura retroesternal foi escolhida por representar os diferentes biótipos de tórax existentes. Apenas a variável ANGCF, por se tratar de um ângulo, não foi normalizada.

\section{Resultados}

Para a correlação entre as variáveis de imagem e de fisioterapia, utilizou-se o coeficiente de correlação de Pearson, avaliando-se sua significância.

Para estimar as médias das variáveis quantitativas da avaliação por imagem e os exames de fisioterapia, considerou-se um intervalo de confiança de 95\%.

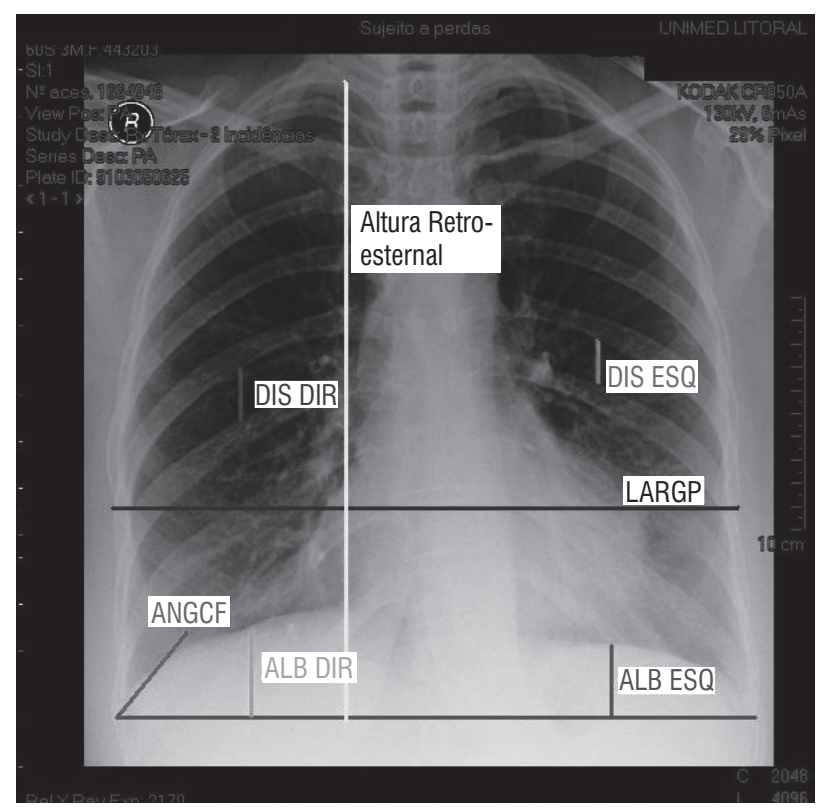

Figura 1- Imagem das medidas analisadas na radiografia Fonte: Dados da pesquisa.
Possibilitou-se, assim, verificar a proximidade entre o parâmetro da população e sua estimativa obtida a partir de uma amostra. Quanto mais estreito for o intervalo de confiança da estimativa calculada, mais próxima ela estará do valor populacional $(18,19)$.

Os dados coletados foram organizados em planilha Excel e analisados com o programa computacional Statística v.8.0 (20) (Tabelas 1 e 2).

Após a coleta e a organização dos dados, eles passaram por análise estatística, apontando-se os resultados que foram analisados e discutidos.

Para cada uma das variáveis de fisioterapia e de imagem, estimou-se o coeficiente de correlação e testou-se a hipótese nula de que não existe correlação, ou seja, o valor de coeficiente de correlação igual a zero, entre as duas variáveis sob análise, versus a hipótese alternativa de que existe correlação (coeficiente de correlação diferente de zero).

Caso o valor de coeficiente assuma valores positivos, isso indica correlação direta (positiva). Sendo assim, valores baixos (altos) de uma variável correspondem a valores baixos (altos) da outra variável. Por outro lado, valores negativos indicam correlação inversa (negativa), ou seja, valores baixos (altos) de uma variável correspondem a valores altos (baixos) da outra.

Na Tabela 3, são apresentados os valores dos coeficientes de correlação estimados e os valores de "p" dos testes estatísticos.

Os dados possibilitaram a identificação de correlação, principalmente, com a variável de imagem ANGCF, que se correlacionou positivamente com as variáveis de espirometria (CVF e PFE) e de cirtometria (Xifoide CR e Axilar CR). Sendo assim, se o valor de ANGCF aumenta, os valores de CVF, PFE, Xifoide CR e Axilar CR também aumentam, e vice-versa. A variável de imagem ALB DIR correlacionou-se positivamente com a variável PI máx. Nesse caso, se o valor do nível de rebaixamento diafragmático (ALB DIR) aumenta, a força muscular inspiratória (PI máx) também, e, caso haja uma redução do ALB DIR, a PI máx, da mesma forma, diminui. O LARGP correlacionou-se negativamente com o ponto verificado pela cirtometria BASAL $\mathrm{CR}$, sendo assim, tem-se uma correlação inversa: caso o LARGP aumente, o BASAL CR diminui, e vice-versa.

As variáveis de imagem ALB ESQ, DIS DIR e DIS ESQ não apresentaram correlação com as variáveis dos exames fisioterápicos. Dos exames, os seguintes dados não apresentaram correlação com as variáveis de imagem: espirometria (VEF1 e VEF1/ CVF), manovacuometria (PE máx) e TC6'. 
Tabela 1- Dados (coletados e normalizados) das radiografias dos indivíduos com DPOC

\begin{tabular}{ccccccc}
\hline PCTE & LARGP & ANGCF & ALB DIR & ALB ESQ & DIS DIR & DIS ESQ \\
\hline 1 & 0,9192 & 42,8800 & 0,0753 & 0,0835 & 0,0733 & 0,0774 \\
2 & 1,0208 & 30,0200 & 0,1896 & 0,1208 & 0,0438 & 0,0396 \\
3 & 1,0413 & 35,3600 & 0,1942 & 0,1359 & 0,0558 & 0,0558 \\
4 & 1,0747 & 39,0900 & 0,0940 & 0,0795 & 0,0723 & 0,0699 \\
5 & 1,1102 & 45,7500 & 0,1916 & 0,1496 & 0,0604 & 0,0604 \\
6 & 0,8598 & 23,6300 & 0,0714 & 0,0438 & 0,0691 & 0,0829 \\
7 & 0,9147 & 41,7300 & 0,1344 & 0,1576 & 0,0904 & 0,0853 \\
8 & 0,8404 & 46,3600 & 0,1155 & 0,0786 & 0,0746 & 0,0693 \\
9 & 0,9181 & 35,3100 & 0,1148 & 0,0932 & 0,0619 & 0,0546 \\
10 & 1,0422 & 40,4500 & 0,0604 & 0,1298 & 0,0687 & 0,0630 \\
11 & 1,1452 & 21,1800 & 0,0676 & 0,1014 & 0,0855 & 0,0895 \\
12 & 1,0195 & 29,3600 & 0,1339 & 0,1166 & 0,0454 & 0,0475 \\
13 & 0,8946 & 26,5700 & 0,0906 & 0,0628 & 0,0739 & 0,0665 \\
14 & 0,9442 & 35,9700 & 0,1759 & 0,1254 & 0,0809 & 0,0849 \\
15 & 0,8700 & 50,1900 & 0,1498 & 0,1282 & 0,0650 & 0,0769 \\
\hline
\end{tabular}

Fonte: Dados da pesquisa.

Legenda: PCTE - paciente; LARGP - largura pulmonar; ANGCF - ângulo costodiafragmático; ALB DIR - nível de rebaixamento diafragmático direito; ALB ESQ - nível de rebaixamento diafragmático esquerdo; DIS DIR - distância intercostal direita; DIS ESQ - distância intercostal esquerda.

Tabela 2 - Dados coletados dos exames de indivíduos com DPOC

\begin{tabular}{ccccccccccccc}
\hline Sexo & Idade & CVF & VEF1 & $\begin{array}{c}\text { VEF1/ } \\
\text { CVF }\end{array}$ & PFE & GOLD & PI máx & PE máx & TC6' & $\begin{array}{c}\text { Axilar } \\
\text { CR }\end{array}$ & $\begin{array}{c}\text { Xifoide } \\
\text { CR }\end{array}$ & $\begin{array}{c}\text { Basal } \\
\text { CR }\end{array}$ \\
\hline F & 54 & 0,5740 & 0,1930 & 0,3300 & 0,2224 & M grave & 45 & 71 & 235,5 & 3 & 1 & 3 \\
M & 70 & 0,6930 & 0,3820 & 0,5500 & 0,4340 & Grave & 102 & 60 & 324,8 & 2 & 3 & -3 \\
M & 72 & 0,8289 & 0,5503 & 0,6639 & 0,7971 & Grave & 67 & 51 & 197,1 & 3 & 2 & -4 \\
F & 60 & 0,8426 & 0,6076 & 0,7211 & 0,7194 & Moderado & 95 & 68 & 473 & 5 & 4 & 3 \\
M & 64 & 0,8274 & 0,5723 & 0,6916 & 0,7742 & Moderado & 72 & 57 & 441,3 & 2 & 5 & -8 \\
F & 75 & 0,7678 & 0,5039 & 0,6500 & 0,6940 & Moderado & 50 & 40 & 480 & 1 & 1 & 1 \\
F & 56 & 1,1313 & 0,4702 & 0,4100 & 0,6694 & Moderado & 84 & 70 & 387 & 3 & 2 & -1 \\
M & 82 & 1,3600 & 0,4732 & 0,3400 & 0,7845 & Grave & 62 & 74 & 250 & 5 & 7 & 10 \\
F & 52 & 0,4900 & 0,3200 & 0,6500 & 0,4650 & Moderado & 67 & 80 & 375 & 3 & 2 & -3 \\
F & 58 & 0,6800 & 0,3300 & 0,5520 & 0,4860 & Grave & 65 & 60 & 278 & 3 & 4 & -2 \\
M & 68 & 0,3612 & 0,1781 & 0,4900 & 0,0970 & M grave & 49 & 98 & 208,8 & 1 & -2 & -1 \\
M & 70 & 0,6970 & 0,3500 & 0,5000 & 0,1730 & Grave & 98 & 101 & 435,6 & 2 & 3 & -3 \\
F & 73 & 0,6700 & 0,2800 & 0,4100 & 0,2200 & M grave & 52 & 84 & 185 & 2 & 1 & -3 \\
M & 69 & 0,6800 & 0,3300 & 0,4800 & 0,5432 & Grave & 90 & 57 & 254 & 1 & 2 & 1 \\
M & 67 & 0,9400 & 0,5400 & 0,5700 & 0,7000 & Moderado & 100 & 93 & 342 & 4 & 2 & 2 \\
\hline
\end{tabular}

Fonte: Dados da pesquisa.

Legenda: F - feminino; $M$ - masculino; CVF - capacidade vital forçada; VEF1 - volume expiratório forçado no primeiro segundo; VEF1/CVF razão de volume expiratório forçado no primeiro segundo sobre a capacidade vital forçada; PFE - pico de fluxo expiratório; GOLD - global initiative for chronic obstructive lung disease; PI máx - pressão inspiratória máxima; PE máx - pressão expiratória máxima; TC6 ' - teste da caminhada de seis minutos; Axilar CR - coeficiente axilar; Xifoide CR - coeficiente xifoide; Basal CR - coeficiente axilar. 
Tabela 3 - Valores dos coeficientes de correlação dos exames fisioterápicos e das variáveis radiográficas

\begin{tabular}{llllllll}
\hline & & LARGP & ANGCF & ALB DIR & ALB ESQ & DIS DIR & DIS ESQ \\
\hline \multirow{2}{*}{ CVF } & Coef. de correlação & $-0,44$ & 0,60 & 0,26 & 0,15 & 0,13 & 0,07 \\
& Valor de $p$ & 0,103 & 0,019 & 0,346 & 0,588 & 0,647 & 0,816 \\
VEF1 & Coef. de correlação & $-0,02$ & 0,44 & 0,45 & 0,20 & $-0,20$ & $-0,12$ \\
& Valor de $p$ & 0,933 & 0,104 & 0,096 & 0,468 & 0,472 & 0,668 \\
VEF1/CVF & Coef. de correlação & 0,44 & $-0,07$ & 0,26 & 0,11 & $-0,41$ & $-0,30$ \\
& Valor de p & 0,102 & 0,805 & 0,355 & 0,702 & 0,127 & 0,283 \\
\multirow{2}{*}{ PFE } & Coef. de correlação & $-0,19$ & 0,57 & 0,42 & 0,22 & 0,00 & 0,03 \\
& Valor de p & 0,503 & 0,028 & 0,117 & 0,428 & 0,994 & 0,910 \\
PI máx & Coef. de correlação & 0,12 & 0,27 & 0,59 & 0,50 & $-0,39$ & $-0,35$ \\
& Valor de p & 0,677 & 0,325 & 0,020 & 0,056 & 0,146 & 0,195 \\
PE máx & Coef. de correlação & 0,04 & $-0,06$ & $-0,22$ & 0,00 & 0,04 & 0,01 \\
& Valor de p & 0,887 & 0,840 & 0,433 & 0,992 & 0,890 & 0,985 \\
\multirow{2}{*}{ TC6' } & Coef. de correlação & 0,05 & 0,08 & 0,05 & 0,00 & $-0,25$ & $-0,12$ \\
& Valor de p & 0,873 & 0,771 & 0,856 & 0,998 & 0,370 & 0,663 \\
\multirow{2}{*}{ Axilar CR } & Coef. de correlação & $-0,21$ & 0,70 & $-0,05$ & $-0,02$ & 0,02 & $-0,12$ \\
& Valor de p & 0,445 & 0,004 & 0,846 & 0,948 & 0,945 & 0,668 \\
\multirow{2}{*}{ Xifoide CR } & Coef. de correlação & $-0,10$ & 0,62 & 0,30 & 0,16 & $-0,29$ & $-0,42$ \\
& Valor de p & 0,733 & 0,013 & 0,283 & 0,568 & 0,301 & 0,115 \\
Basal CR & Coef. de correlação & $-0,54$ & 0,29 & $-0,37$ & $-0,46$ & 0,41 & 0,43 \\
& Valor de p & 0,038 & 0,301 & 0,179 & 0,083 & 0,133 & 0,111 \\
\hline
\end{tabular}

Fonte: Dados da pesquisa.

Legenda: LARGP - largura pulmonar; ANGCF - ângulo costodiafragmático; ALB DIR - altura da base do diafragma direito; ALB ESQ - altura da base do diafragma esquerdo; DIS DIR - distância intercostal direita; DIS ESQ - distância intercostal esquerda. CVF - capacidade vital forçada; VEF1 - volume expiratório forçado no primeiro segundo; PFE - pico de fluxo expiratório; PI máx - pressão inspiratória máxima; PE máx - pressão expiratória máxima; TC6' - teste de caminhada de seis minutos; Axilar CR - coeficiente axilar; Xifoide CR - coeficiente xifoide; Basal CR - coeficiente axilar.

\section{Discussão}

O ponto analisado para a largura pulmonar (LARGP) apresentou correlação com o exame de cirtometria para o ponto Basal, que se refere à mobilidade na região abdominal durante a respiração.

A presença do tórax em barril, em virtude da hiperinsuflação, faz com que ocorra a elevação do gradil costal e a projeção das costelas para frente. A mudança na conformação anatômica do tórax reflete na participação da musculatura abdominal na respiração, já que esta fica limitada por um tórax hiperinsuflado.

Quando na expiração, tem-se a contração do diafragma, que empurra as vísceras abdominais contra a parede abdominal. Os músculos abdominais, por sua vez, contraem-se e empurram o diafragma relaxado para o interior da caixa torácica, tendo-se, assim, a expiração. Na DPOC, esse movimento está limitado pelas alterações na caixa torácica e pelomovimento do diafragma; sendo assim, a movimentação dos músculos abdominais também será alterada $(21,5)$.

0 variável que teve maior correlação com os exames de fisioterapia foi a ANGCF, que se correlacionou com variáveis de espirometria (CVF e a PFE) e de cirtometria (Axilar CR e Xifoide CR). A variável ANGCF está relacionada ao diafragma, que, por sua vez, tem na hiperinsuflação a causa para suas alterações de posicionamento.

Observou-se, no estudo, que a correlação da CVF e do ANGCF é positiva. Considera-se que a CVF é um indicador da capacidade de mobilizar volume pulmonar, o que depende da ação do diafragma. Sendo assim, proporcionalmente, tendo um ângulo de 
diafragma maior, maior será a CVF e a capacidade de mobilizar volume.

Na DPOC, essa capacidade encontra-se diminuída, assim como o ângulo do diafragma, indicando um rebaixamento de suas hemicúpulas, em decorrência da presença de inflamação sistêmica muscular e de hiperinsuflação. A destruição das fibras elásticas provocada pelo enfisema gera alterações na caixa torácica e nos valores obtidos por meio da espirometria (10).

Já a variável PFE está ligada a alterações em vias aéreas proximais e à diminuição do volume pulmonar. Sua medição é de valia para doenças restritivas como a asma, porém isoladamente não é um indicativo de doenças obstrutivas como a DPOC (10).

A correlação entre o PFE e a variável ANGCF encontrada neste estudo foi inesperada, já que o valor de PFE não é característico de alteração na DPOC e reflete a força muscular expiratória; já o ANGCF verifica o posicionamento do diafragma, que é um músculo classificado como inspiratório.

A característica do diafragma é participar da inspiração, momento em que realiza contração. Porém, ele atua também na expiração, quando seu tônus é mantido, ocasionando uma ação de frenagem do movimento $(21,22)$.

Durante a expiração, o diafragma relaxado é empurrado superiormente pelas vísceras abdominais, desinsuflando o pulmão. Na ocorrência de alteração da mecânica do diafragma, ressalta-se que ele se encontra com sua incursão diminuída, limitando a ação do conteúdo abdominal. Assim, sua capacidade de auxiliar na expiração está prejudicada, o que poderia apontar a correlação com o PFE (23).

Para avaliação da mobilidade torácica, foi utilizada a cirtometria. As consequências da hiperinsuflação são perceptíveis na mobilidade torácica e também na alteração da posição anatômica do diafragma, por isso houve correlação entre o ponto axilar e o xifoide com a variável de imagem ANGCF.

As variáveis de imagem tiveram maior correlação com o exame de cirtometria, que avalia a expansibilidade torácica. Embora o valor de cirtometria utilizado nesta pesquisa tenha sido do coeficiente entre a inspiração e a expiração, uma manobra dinâmica, ao passo que a avaliação do tórax é estática, o exame de radiografia é executado no momento de uma inspiração. Utiliza-se, então, para esses dois exames, uma mesma manobra respiratória, o que explicaria a presença de tal correlação.
A hiperinsuflação leva a alterações estruturais dos músculos da caixa torácica. 0 diafragma, devido à hiperinsuflação, retifica-se e tem sua incursão limitada (7).

Quando um tórax apresenta sua capacidade de expansibilidade reduzida pela presença da DPOC, a mobilidade torácica avaliada pela cirtometria é alterada, bem como a disposição do diafragma. Seu ângulo estará diminuído, pois tal alteração modifica a função pulmonar, não permitindo uma expansão torácica efetiva.

No estudo realizado por Yamaguti (24), observou-se que indivíduos com DPOC que apresentam disfunção diafragmática definida por baixa mobilidade do diafragma possuem maior risco de mortalidade, quando comparados aos indivíduos sem disfunção diafragmática. Isso indica que uma mobilidade diafragmática reduzida associa-se com maior gravidade da doença.

A ALBDIR é outra variável de imagem que se correlacionou com a variável de PI máx avaliada pela manovacuometria. Essa correlação é perceptível, já que o diafragma é um músculo inspiratório e sua força é mensurada pela PI máx, e a variável ALBDIR indica a disposição do diafragma após uma inspiração (solicitada para a realização da radiografia) diante da presença de hiperinsuflação. Sendo assim, com o diafragma retificado, o valor de ALB DIR correlaciona-se com a força que esse músculo é capaz de produzir diante das alterações provocadas pela DPOC.

O rebaixamento das cúpulas diafragmáticas leva o músculo a trabalhar com uma carga maior e reduz o comprimento das fibras musculares; assim, o diafragma não gera a força necessária para realizar o movimento $(6,23)$.

A mesma correlação não foi observada em relação à ALB ESQ. Isso pode ocorrer pois, nesse hemitórax, o posicionamento do diafragma sofre influência da presença da área cardíaca, fator que já altera a posição do diafragma no tórax de indivíduos normais e, também, em indivíduos com DPOC, principalmente quando apresentam doenças cardíacas associadas.

Para a musculatura expiratória avaliada pela PE máx, não foi estabelecida nenhuma correlação com as variáveis da imagem. Isso pode ter ocorrido já que a expiração é uma manobra passiva, sendo os músculos expiratórios solicitados apenas em atividade física, quando a respiração se torna ativa.

As variáveis de imagem ALB ESQ, DIS DIR e DIS ESQ não obtiveram correlação com os exames de fisioterapia. A não correlação das distâncias intercostais 
direita e esquerda com nenhum dos exames corrobora com estudo realizado por Walsh et al. (25). Analisando as alterações da disposição das costelas em indivíduos normais e com DPOC, os autores verificaram que elas não apresentaram alterações estruturais significativas. 0 estudo ainda cita que a principal alteração estrutural do tórax em pacientes com DPOC com hiperinsuflação crônica limita-se ao diafragma.

Da mesma forma, o VEF1 e a relação VEF1-CVF não apresentaram correlação com as variáveis radiográficas. Essas variáveis da espirometria são indicativas de obstrução ao fluxo aéreo e padrão ouro no diagnóstico da DPOC. Porém, tais dados isolados não refletem a magnitude das manifestações sistêmicas ocasionadas pela DPOC no indivíduo.

Sendo assim, tem-se a necessidade de traçar novos marcadores de gravidade e prognóstico da DPOC. Gold (26) aponta que, para um diagnóstico diferencial da DPOC, é necessário avaliar o histórico do cliente e os exames laboratoriais e de radiografia (26).

O VEF1 é padrão ouro para diagnóstico e estadiamento da DPOC, avaliado pela espirometria. Ainda é um marcador importante, entretanto, são necessárias avaliações adicionais para estimar, de forma mais completa, todas as repercussões da doença (27).

0 teste de caminhada de seis minutos foi outra variável de exame fisioterápico que não apresentou correlação com as variáveis de imagem. Esse é um teste submáximo que avalia a capacidade física aeróbica do indivíduo, sendo um indicador de qualidade de vida e funcionalidade $(11,28)$.

Na pesquisa, o TC6' não apresentou correlação estatística com nenhuma variável de imagem. Isso pode ser explicado, já que a capacidade física aeróbica evidenciada pelo TC6' é dependente do débito cardíaco e da diferença arteriovenosa de oxigênio, não existindo correlação com a conformidade anatômica.

Em pesquisa desenvolvida por Paulin (29) com exercícios para incremento da mobilidade torácica, ocorreu uma melhora no teste de caminhada após a aplicação de protocolo de exercícios para mobilidade. Associou-se essa correlação com a ventilação pulmonar, em função de ganho da capacidade vital forçada ou pela melhora do padrão respiratório. Porém, não se acredita que houve alteração no metabolismo aeróbico muscular, o que justificaria uma correlação com o TC6' (29).

Tal fato pode indicar que as alterações de caixa torácica não influenciam a capacidade de o indivíduo realizar as atividades de vida diária. Sendo assim, independentemente do impacto da DPOC na caixa torácica, pode-se obter melhora em relação à qualidade de vida quando o individuo é submetido a treinamento aeróbico, o que melhora sua endurance e sua capacidade de executar exercícios $(29,30)$.

\section{Conclusão}

A variável de imagem que mais se correlacionou com os exames de fisioterapia foi a ANGCF. Os resultados mais expressivos foram observados com variáveis de exames de espirometria e cirtometria, indicando que a posição do diafragma impacta na função pulmonar e na mobilidade torácica. A LARGP correlacionou-se com a espirometria e a cirtometria, e a ALB, com a manovacuometria.

O uso de ferramentas tecnológicas para auxílio no diagnóstico possibilita obter dados quantitativos das alterações patológicas. Quando se fala em DPOC, uma doença que leva a alterações sistêmicas, a avaliação dessas alterações possibilita dilatar o conhecimento de seu impacto na vida do indivíduo.

Para a fisioterapia, essa avaliação contribui para uma compreensão ampliada das alterações musculoesqueléticas, o que pode auxiliar na escolha da intervenção fisioterápica.

\section{Agradecimentos}

Ao profissionais que atuam no Complexo de Medicina Preventiva Unisaúde e na Clínica de Fisioterapia da Univali, em Itajaí (SC), por possibilitarem a realização do estudo em suas dependências.

\section{Referências}

1. Sociedade Brasileira de Pneumologia e Tisiologia. II Consenso brasileiro sobre doença pulmonar obstrutiva crônica - DPOC. J Pneumol. 2004; 30 suppl 5.

2. O'Donnell DE, Aaron S, Bourbeau J, Hernandez P, Marciniuk DD, Balter M, et al. Canadian thoracic society recommendations for management of chronic obstructive pulmonary disease. Can Respir J. 2007;Suppl B:5B-32B.

3. Fu WP, Zhao ZH, Fang LZ, Sun C, Liu L, Zhang JQ et al. Heme oxygenase-1 polymorphism associated with severity of chronic obstructive pulmonary disease. Chin Med J. 2007;120(1):12-6. 
4. Sullivan, DC. Imaging as a quantitative science. Radiology. 2008;248(2):328-32.

5. Scanlan CL, Wilkins RL, Stoller JK. Fundamentos da terapia respiratória de Egan. 7a ed. Rio de Janeiro: Manole; 2000

6. Dourado VZ, Tanni SE, Vale SA, Faganello MM, Sanchez FF, Godoy I. Manifestações sistêmicas na doença pulmonar obstrutiva crônica. J Bras Pneumol. 2006;32 (2):161-72.

7. Global Iniciative For Chronic Obstructive Lung Disease GOLD. Global strategy for the diagnosis, management, and prevention of chronic obstructive pulmonary disease NHLBI/WHO. Workshop Report, 2008; [acesso 25 jul. 2010]. Disponível em http://www.goldcopd.com/Guidelineitem.asp?l1=2\&l2=1\&intId=989.

8. Li Q Li F, Suzuki K, Shiraishi J, Abe H, Engelmann R, et al. Computer-aided diagnosis in thoracic CT. Semin Ultrasound CT MR. 2005;26(5):357-63.

9. American Toracic Society. Standardization of spirometry. Am J Respir Crit Care Med. 1995;152:1107-36.

10. Pereira CAC. Diretrizes para teste de função pulmonar. J Pneumol. 2002;28(Suppl 3).

11. American Toracic Society. Statement guidelines for the six-minute walk test. Am J Respir Crit Care Med. 2002;166(1):111-7.

12. American Colle Sports Medicine. Manual para teste de esforço e prescrição de exercício. 4a ed. Rio de Janeiro: Shape; 1999.

13. Caldeira, VS, Starling CCD, Britto RR, Martins JA, Sampaio RF, Parreira VF, et al. Precisão e acurácia em adultos saudáveis. J Bras Pneumol. 2007; 33(5):519-26.

14. Gracia RCP, Costa D. Treinamento muscular respiratório em pós-operatório de cirurgia cardíaca eletiva. Rev Bras Fisiot. 2002;6(3):139-46.

15. Black LF, Hyatt RE. Maximal respiratory pressures: normal values and relationship to age and sex. Am Rev Respir Dis. 1969;99(5):696-702.

16. Sousa RB. Pressões respiratórias estáticas máximas. J Pneumol. 2002;28 Suppl 3:S155-65.

17. Brunetto AF, Alves LA. Comparação entre os valores de pico sustentados das pressões respiratórias máximas em indivíduos saudáveis e pacientes portadores de pneumopatia crônica. J Pneumol. 2003;29(4):208-12.

18. Callegari-Jacques SM. Bioestatística: princípios e aplicações. Porto Alegre: Artmed; 2003.
19. Vieira S. Introdução a bioestatística. Rio de Janeiro: Campus; 1980.

20. Arango HG. Bioestatística: teórica e computacional. 2a ed. Rio de Janeiro: Guanabara Koogan; 2005.

21. West JB. Fisiologia Respiratória. 6a ed. Barueri: Manole; 2002.

22. Levitzky MG. Fisiologia Pulmonar. 6a ed. Barueri: Manole; 2004.

23. Kisner C, Colby LA. Exercícios terapêuticos: fundamentos e técnicas. 4a ed. Rio de Janeiro: Manole; 2004.

24. Yamaguti WPS, Paulin E, Salge JM, Chammas MC, Cukier A, Carvalho CRF. Disfunção diafragmática e mortalidade em pacientes portadores de DPOC. J Bras Pneumol. 2009;35(12):1174-81.

25. Walsh JM, Webber CL Jr, Fahey PJ, Sharp JT. Structural change of the thorax in chronic obstructive pulmonary disease. J Appl Physiol. 1992;72(4):1270-8.

26. Global Iniciative for Chronic Obstructive Lung Disease GOLD. Global strategy for the diagnosis, management, and prevention of chronic obstructive pulmonary disease: update 2009; [acesso 28 jul. 2010]. Disponível em: http://www.goldcopd.com/download.asp?intId=554.

27. Godoy I. Avaliando a gravidade e o prognóstico da doença pulmonar obstrutiva crônica: a medida do VEF1 ainda é suficiente? J Bras Pneumol. 2007;33(4):23-4.

28. Rondelli RR, Oliveira AN, Dal Corso S, Malaguti C. Uma atualização e proposta de padronização do teste de caminhada de seis minutos. Fisioter Mov. 2009;22(2):249-59.

29. Paulin E. Efeitos de um programa de exercício físico direcionado a mobilidade torácia na capacidade funcional e psicossocial em pacientes portadores de DPOC [dissertação]. São Paulo: Faculdade de Medicina da Universidade de São Paulo; 2000.

30. Pitta F, Troosters T, Probst VS, Lucas S, Decramer M, Gosselink R. Possiveis consequências de não se atingir a mínima atividade física diária recomendada em pacientes com doença pulmonar obstrutiva crônica estável. J Bras Pneumol. 2006;32(4):301-8.

Recebido: 05/09/2011 Received: 09/05/2011

Aprovado: 23/04/2012 Approved: 04/23/2012 